\title{
2018 Canadian Urological Association guideline for Peyronie's disease and congenital penile curvature
}

Anthony J. Bella ${ }^{1}$; Jay C. Lee ${ }^{2}$; Ethan D. Grober ${ }^{3}$; Serge Carrier ${ }^{4}$; Francois Benard ${ }^{5}$; Gerald B. Brock ${ }^{6}$

${ }^{1}$ Urology and Men's Health, Ottawa Hospital Research Institute, University of Ottawa, Ottawa ON; ${ }^{2}$ Department of Surgery, Division of Urology, University of Calgary, Calgary, AB; ${ }^{3}$ Division of Urology, Department of Surgery, University of Toronto, Toronto, ON; ${ }^{4}$ Division of Urology, McGill University Health Centre, Montreal, QC; ${ }^{5}$ Department of Surgery, Université de Montreal, Montreal, QC; ${ }^{6}$ Department of Surgery, Division of Urology Western University, London, ON; Canada

Cite as: Can Urol Assoc J 2018 Feb. 22; Epub ahead of print. http://dx.doi.org/10.5489/cuaj.5255

Published online February 22, 2018

$* * *$

\section{Introduction}

Peyronie's disease (PD) is a highly prevalent condition which affects the physical and psychosocial well-being and quality of life (QoL) for thousands of Canadian men. The specific etiology of PD remains poorly understood and there remains a paucity of randomized placebocontrolled trials evaluating treatment interventions. ${ }^{1-3} \mathrm{PD}$ can be found in up to $8.9 \%$ of men, a remarkable increase in cited prevalence that is attributable to growing awareness (as historical data suggested a rate of less than 1\%); the burden of disease is significant, and PD is often present in otherwise healthy men. The following Guidelines were crafted by the committee with a full awareness of the limitations of the literature, and sought to provide actionable recommendations to guide PD care in the Canadian health system.

\section{Natural history}

Peyronie's disease is an acquired penile disorder characterized by benign fibrotic changes primarily to the tunica albuginea (TA), resulting in a constellation of signs and symptoms occurring alone or in combination, including penile deformity (curvature, narrowing, indentation, hinging), erectile dysfunction, penile pain, shortening and plaque formation. ${ }^{4}$ The presence and severity of these symptoms, as well as the timing of the presentation for medical evaluation is variable, as is the degree of morbidity and impact on sexual function. The natural history of PD is not that of improvement/and or resolution of the features of the condition. The largest series (Mulhall et al, 2006) ${ }^{5}$ to report on the natural history of untreated patients with PD included 246 patients with at least 1 year follow up. Patients' baseline pain improved in all men, with complete pain resolution documented in $89 \%$ of patients. In contrast, penile curvature was only 
reported to improve in $12 \%$ of untreated men with the condition. Penile curvature worsened for $48 \%$ and remained stable in $40 \%$ of men. More recently in a smaller series of 176 patients, Berookhim et al. reported curvature improvement in 12\%, worsening in $21 \%$ and unchanged curvature in $76 \%$ of untreated patients followed for at least 12 months. ${ }^{6}$ Natural history studies are influenced by the duration of follow up. Over the longest reported observation period, Grasso et al. studied 110 men followed for at least 5 years (mean follow up 6.4 years). ${ }^{7}$ In contrast to reports of shorter follow up durations, disease progression was more common with $68 \%$ of men $<50$ years of age requiring surgical therapy and 31.5\% of men $>50$ years of age opting for surgical treatment.

Collectively, the natural history studies related to Peyronie's disease suggest that plaquerelated pain improves and/or resolves in the majority of patients with time even in the absence of treatment. ${ }^{1,3-7}$ Conversely, spontaneous resolution or significant improvement of penile deformity is rare, and predictors of disease progression/resolution are inconsistent or absent. ${ }^{6,7}$ As such patients with penile deformities that result in sexual dysfunction or distress should be counseled regarding treatment options that may influence the natural history of the condition and restore functionality.

\section{Peyronie's disease and QoL impact}

The relationship between PD and erectile dysfunction due to resultant physical penile deformity is clear, as are most diagnoses of PD. In the clinical practice setting, a diagnosis of erectile dysfunction (ED) or failed first- and second-line ED treatments warrants ruling out PD. Many cases of PD are initially treated unsuccessfully as ED without identifying and addressing the penile deformity. ${ }^{8}$ A careful history may identify the impact of deformity on erectile function or the presence of a flail segment (satisfactory rigidity present in the penis proximal to the plaque(s)). The presence of penile pain, especially with erection, increases the likelihood of a penile septal scar, an atypical form of PD which may be characterized by symptoms including penile shortening, pain, and ED, even in the absence of deformity or palpable disease. ${ }^{9-10}$ Awareness of psychosocial distress has been classically under-represented in the literature and in practice. ${ }^{11}$ Numerous studies have now demonstrated significant distress in patients suffering with PD as well as their partners (both heterosexual and same sex partnerships). ${ }^{12-14}$ Other studies have demonstrated significant findings on depression scales, with Nelson et al reporting that $47 \%$ of men with PD reported clinically meaningful depression. ${ }^{15-16}$ Men with PD report embarrassment and shame, and may avoid sexual relationships; this may take a tremendous toll on their quality of life. ${ }^{11}$ Severity of the PD as well as inability to have sex, can increase the distress experienced by the patient. ${ }^{17-18}$ Clinicians should be aware and identify the degree of bother and distress experienced by the patient and their partner. Patients should be offered psychological support. ${ }^{19}$ Rosen et al identified six main areas of concern among men with PD: physical appearance, sexual self-image, loss of sexual self-confidence and attractiveness, sexual function and performance, performance anxiety, and social stigmatization. ${ }^{11}$ Many of the men 
with PD expressed a sense of social isolation, and found it difficult to communicate with healthcare professionals or sexual partners; the clinician's awareness of this may help elucidate a more detailed patient history and QoL impact. Use of PD questionnaires help to identify the psychosocial distress, and have demonstrated that successful improvement in PD can improve psychosocial outcomes. ${ }^{20-21}$ Smith et al reported that $81 \%$ of 245 PD patients reported emotional difficulties and 54\% reported relationship problems as a result of PD. ${ }^{14}$ On multivariate analysis, the presence of relationship problems and loss of penile length were independent predictors of emotional problems due to PD.

\section{Methods}

The Canadian Urological Association Guidelines Committee sought to provide guidelines for the management of Peyronie's disease (PD) and Congenital Penile Curvature (CPC) at the point of care utilizing a Canadian perspective. Suggestions for management were based on the peer reviewed literature, the 4th International Consultation on Sexual Medicine (June 2015), International Society for Sexual Medicine Guidelines, American Urological Association Guidelines, and Sexual Medicine Society of North America PD management recommendations. A comprehensive literature search was performed using Pubmed, Cochrane and EMBASE, with data cutoff at 6/30/2017. Prospective or retrospective studies, as well as review studies providing data for the natural history, pathophysiology, diagnosis and/or management of PD and separately, congenital penile curvature (CPC) were included; preclinical studies were excluded. As performed for other contemporary CUA guidelines, such as those for cystic renal lesions in $2017,{ }^{22}$ the International Consultation for Urologic Disease (ICUD)/World Health Organization (WHO) modified Oxford Center for Evidence-Based Medicine grading system was used to grade each topic assessed, and the level of evidence summarized as follows: Level 1 - meta-analysis of randomized controlled trials (RCTs) or good quality RCT; Level 2 - low quality RCT or metaanalysis of good quality prospective cohort studies; Level 3 - good quality retrospective casecontrol studies or case series; Level 4 - expert opinion. Dahm and colleagues have eloquently critiqued the 2015 ICUD methodology used by Chung et al, and the American Urological Association PD guidelines have utilized "linking type to evidence stength" with resultant Strong, Moderate, and Conditional (non-directive) recommendations. ${ }^{1-3}$ The committee has performed an expert review of the literature and this document represents a consensus of all co-authors of these guidelines, basing recommendations as Grade A: consistent with Level 1 evidence; Grade B: consistent with Level 2 or 3 evidence; Grade C: majority proportion of evidence from Level 2 or 3 studies or level 4 evidence; and Grade D; no recommendation possible based on incomplete evidence or consensus expert opinion. The low quality of evidence in the PD literature made it difficult to make Grade A or B recommendations, and the committee did its best to summarize the current literature and provide usable guidance for the management of PD and CPC. It is the hope of the group that data published after the literature search cut-off is of rigor that will allow for incorporation into the next version of these guidelines. 


\section{Section one: Diagnosis}

\section{Overview}

The CUA supports the view that the general framework for the evaluation of patients with any type of sexual dysfunction should follow the accepted basic principles. ${ }^{8}$ The sexual history should ascertain the severity, onset, and duration of the problem, concurrent or contributory medical or psychosocial factors, and bother to the patient and partner (if applicable). The inperson interview is often supplemented with disease- or problem specific questionnaires outlined in this section. Given the emotional and psychological impact of PD and sexual dysfunction, the tone of the sexual inquiry is important and should reflect a high level of sensitivity and regard for each individual's unique ethnic, cultural, and personal background.

\section{Patient evaluation}

There are no randomized trials evaluating the diagnosis or assessment of PD. All recommendation for diagnosis and assessment are based on Clinical Principles and Expert Opinion, and provide clinicians and patients with a framework for determining the diagnosis of PD (Level 4 evidence, Grade C Recommendation). ${ }^{1,3,22-23}$ Diagnosis is based on patient history and physical examination, including patient-provided images of the penis at best possible erection. Penile color duplex ultrasonography, if available, provides a safe, low-cost means objective characterization of PD. Intracavernous injection (ICI) of vasoactive agent is the gold standard prior to invasive interventions; if the patient cannot provide digital images at full rigidity (i.e. attain an erection sufficient to characterize the extent of deformity on his own or with the aid of phosphodiesterase-5 inhibitors), ICI is recommended.

\section{PD specific evaluation should include:}

History. Data include onset, duration, history of traumatic event if applicable, deformity and erectile changes over time, acquired vs lifelong (to differentiate PD vs. Congenital Penile Curvature), and medical history inclusive of family presence of PD, Dupuytren's contracture, ${ }^{24}$ and other related conditions that may impact erectile and sexual function. Prior PD and ED treatments should be documented.

Penile characteristics. It is critical to determine the extent of penile deformity, direction of curvature, presence of hourglass deformity, palpable plaque(s), interference with intercourse, penile pain with and with erection, shortening, quality of penile rigidity, and presence of hinging. Penile sensation, ejaculatory function, and length/girth concerns should be documented.Digital home photographic documentation may aid in objectively determining treatment effects, especially when non-surgical options are utilized.

Sexual function. Erectile rigidity, ability to penetrate, and ability to complete intercourse, partner complaints and support. The use of the International Index of Erectile Function - 5 (IIEF5) may of use, as may the Disease Questionnaire Peyronie's (PDQ) which is a newer 15-item validated instrument specific to $\mathrm{PD} .^{20,21}$ The PDQ is responsive to changes in symptoms and 
disease progression, measuring severity in the three domains of physical/psychological symptoms, penile pain, and symptom bother (Level 3 Evidence, Grade C Recommendation). Should bother/psychologic impact warrant, consideration may be made for referral to a mental health professional with expertise in sexuality.

\section{Physical examination}

The penis should be examined on the stretch. Palpation should be performed to identify the location, size, number and tenderness of the plaque. Stretched penile length can also be determined as penile length loss is a primary concern and contributor to distress for PD patients; this is measured from the penopubic skin junction to the coronal sulcus or the tip of the penis. ${ }^{1,3}$ The presence of multiple plaques on both sides of the penis, or plaques within the intracavernosal septum can result in penile shortening with or without deformity. ${ }^{9,25}$

Patient self-reporting of curvature versus objective examination supports the clinical principal that prior to non-invasive or invasive treatments (intralesional injection therapy or surgery), and to monitor treatment effect, it is recommended to examine the erection to determine penile length, degree of curvature, hourglass deformities, and rigidity of erection. ${ }^{1-3}$ Digital photographs at full erection are measured via protractor to determine angle(s) of uni- and multiplanar disease. Examination after injection of penile injection after vasoactive injection in the office remains the gold standard, especially for the patient reporting complex deformity (hourglass or bidirectional curvature) or ED.

\section{Investigations}

An accurate appraisal of the deformity includes identifying the type(s) of deformity, assessing the magnitude or severity, and evaluating penile stability/buckling. The most reliable means of assessing deformity and accomplishing all three goals of assessment is the use of intracavernosal injections, with or without color duplex ultrasonography (CDU).

Penile color doppler ultrasonography (CDU)may be offered (Level 4 Evidence, Grade C Recommendation). This may not be readily be available nor frequently performed in some centers. Doppler may identify tunical thickening and/or calcification and septal plaques; ${ }^{26}$ in a series of 95 patients, Bekos et al reported that the natural history of Peyronie's disease could be predicted based on baseline ultrasonographic plaque characteristics. Solitary, less calcified and dense plaques were predictors of future disease stability as compared to multiple, dense calcified plaques which portend a poorer prognosis. ${ }^{27}$ Combination of ultrasound with intracavernosal injection may also identify arterial insufficiency or veno-occlusive dysfunction, influencing choice of PD management. $^{3}$

High-resolution T2 Magnetic Resonance Imaging (MRI) without fat suppression, has been shown to be an excellent imaging modality for penile pathology including PD but its routine use in clinical practice is not supported; ${ }^{4}$ similarly computed tomography and plain radiography do not have a role routine PD evaluation. Given the lack of specific findings related 
to PD, routine laboratory testing is not recommended, rather targeted bloodwork may be obtained in response to specific findings on history or physical examination (for example, signs/symptoms of hypogonadism). ${ }^{1,3}$

\section{Section two: Non-surgical management of PD}

It is essential to recognize that PD is a symptom complex that may compromise sexual function and quality of life, may affect men from early decades of life through their later years, and does not have a clearly defined management pathway due to the heterogeneity of the disease itself. The patient should be aware that not all urologists have the training, experience, and resources to conduct full evaluation, counsel on various treatment options, and offer care oriented to patient PD and goals. It is entirely appropriate for urologist-to-urologist referral, as within the Canadian health care context not all regions will maintain PD expertise and a goal such as this elusive given systemic demands and constraints.

\section{Clinical principle}

Clinicians should discuss the various aspects of a potential treatment plan, including careful weighing of potential benefit to the patient versus adverse events. As PD does not impact survival, for some men, thoughtful review and counselling regarding their PD, impact on QoL, disease course, and management options may constitute their "treatment". As there is no minimum criteria for deformity necessary for management, a patient may decide to seek treatment based on distress over symptoms, penile appearance, and penile function, which for another would constitute end-of-treatment success or PD not requiring any intervention. For most men, deformity less than 30 degrees does not impair function; the Committee supports a clear discussion with the patient of their PD after evaluation and integration of treatment choices into their care plan which is consistent with patient symptom status, current health, and treatment goals. $1,3,23$

\section{Oral and topical therapies}

\section{Oral therapy}

There is currently no approved oral treatment of Peyronie's disease in Canada. The few available trials have not enrolled enough patients to attain sufficient power, and meta-analysis is difficult because of the heterogeneity of treatments and duration of follow-up, as well as inconsistencies across study endpoints.

The following medications have either been shown in studies with low/moderate level of evidence to be without proven efficacy/limited potential efficacy and may have deleterious side effects.

None of the following oral agents are recommended for standard care of PD in Canada: Vitamin E, tamoxifen, procarbazine, and Vitamin E/L-carnitine are not used in the treatment of PD. Vitamin E in particular does not have any evidence for efficacy. The oral agents 
potassium para-aminobenzoate, colchicine, co-enzyme Q10, and/or pentoxifylline may be considered for clinical use, alone or as a part of multimodal care (oral, intralesional, and traction therapies) but there are clear limitations to the evidence; the American Urological Association has identified these agents as possibly promising but with insufficient evidence to support even a Conditional Recommendation for use until a larger or more rigorous evidence base is avaialable. ${ }^{1,3}$ It is the consensus of this panel that these oral agents may be offered as part of PD care, recognizing limitations to efficacy data, alone or as part of multimodal care. Care should be taken not to unnecessarily postpone other PD therapies, and limitations to evidence and added patient-borne costs of treatment should be clearly communicated (Level 3 Evidence, Grade C Recommendation). The use of PDE-5 inhibitors, specifically tadalafil $5 \mathrm{mg}$ OD to modify Peyronie's plaque progression appears promising, but to date data is limited to a single published study. $^{25}$

Current medical literature is replete with several further agents proposed solely on their efficacy in animal models of PD, when in fact there is valid concern that such models are not representative of human $\mathrm{PD}$. Use in a study setting with full patient consent or in special situations may be justified on an ad hoc basis, but clearly the evidence is simply not in place for general use.

Oral non-steroidal anti-inflammatory medication may be used to control the pain associated with the inflammation during the active phase of the disease. Penile pain may confer significant distress and may compromise sexual function; the ideal agent, duration, and re-assessment has not been elucidated. 1,3

The treatment of erectile dysfunction concomitant with Peyronie's disease follows CUA guidelines for the management of erectile dysfunction. ${ }^{8}$ Oral PDE-5 inhibitors are utilized in patients for whom there are no medication-specific contraindications; if the degree of deformity makes penetrative intercourse difficult due to PD angulation, the patient (and partner) should minimize pain and potential injury by limiting positions to those allowing comfortable penetration.

2. Topical electromotive therapy (Iontophoresis) with verapamil or dexamethasone

The use of iontophoresis is not recommended. There remains an absence of convincing efficacy and a substantial burden of administration. The CUA position is consistent with both the recent evaluations by the AUA and the ICSM(Level 4 Evidence, Grade 3 Recommendation).., 1,28,29

\section{Topical therapy - verapamil gel}

Uncertain and only potential efficacy is seen with the use of verapamil gel and its use cannot be supported by the Committee with the current levels of evidence, as only a single small study exists (less than 20 patients) (Level 4 Evidence, Grade 3 Recommendation). Martin et al demonstrated that topical verapamil fails to infiltrate the tunica albuginea (TA) given the TA's physical properties. ${ }^{30}$ Interestingly, in an earlier double-blinded study by Fitch et al., 18 men 
with PD were randomized to topical verapamil vs. placebo and at 3 months, $61 \%$ reported a decrease in penile curvature and $88 \%$ reported resolution of penile pain. Despite reported improvements in curvature, objective pre-treatment and post-treatment curvature measurement was not performed which limits these findings; ${ }^{31}$ a better quality randomized design of sufficient size executed to contemporary objective endpoints is required before a recommendation for verapamil gel as a PD treatment can be made. ${ }^{3}$

\section{Intralesional therapies}

Intralesional verapamil (ILV) is a widely used local agent for PD treatment in Canada. It is the opinion of this panel that select intralesional therapies can be offered as treatment options for PD; local therapeutic approaches for Peyronie's diseaseis an appealing prospect, potentially obviating the need and risk of surgical management.Intralesional injection has the advantage of rapid and direct local delivery of the active agent into the target tissue, theoretically without the risk of systemic side effects and the potential for achieving high local concentrations.A thorough review of the existing world literature on this approach, unfortunately yields disappointing results. ${ }^{1,3,32}$ While regulatory approval has been granted to purified clostridial collagenase (Xiaflex $^{\mathrm{TM}}$ ) in some markets (Grade B evidence), most injectable agents used for PD are offlabel including all currently utilized agents in Canada. A wealth of single-center reports with relatively small numbers of subjects and variable outcome measures are the norm for intralesional therapy in PD, making comparison and assessment of true efficacy, best approach and realistic likelihood of positive outcomes difficult to determine. Among the greatest challenges to the reader of this literature, is the lack of standardized outcome measurements, choice of and dose of agent, frequency of delivery and patient selection criteria to optimize results.

The committee recommends the use of intralesional verapamil for the management of Peyronie's disease in Canada (Level 3 Evidence, Grade C Recommendation), and anticipates regulatory approval of clostridial collagenase prior to the next revision of these guidelines. A full evaluation and analysis for Xiaflex ${ }^{\mathrm{TM}}$ will be considered in the interim as a Guidelines update.

\section{Collagenase (Xiaflex ${ }^{\mathrm{TM}}$ )}

Collagenases are enzymes able to degrade interstitial collagens. In the United States, the Food and Drug Administration approved Xiaflex (collagenase clostridium histolyticum) for use in PD; as of July 2017 , at the time of writing, it is not approved in Canada for the treatment of PD but we have an expectation of approval in 2018 . Use of collagenase for PD was first described by Gelbard in 1982, in which collagenase was injected into PD plaques removed from patients and studied in vitro. ${ }^{33}$ In 1985 , Gelbard performed the first human trials. ${ }^{34}$ Intralesional collagenase injection of clostridial collagenase has demonstrated true efficacy with a significant decrease in penile curvature, plaque size and an improvement in PD symptom bother scores in multiple trials. Two large multi-center placebo controlled prospective and randomised trials (IMPRESS I \& II) ${ }^{35}$ have identified optimal patients for this technique of intralesional 
collagenase with modelling to be: stable disease with a curvature greater than $30^{\circ}$ and less than $90^{\circ}$, no isolated hourglass deformity or calcified plaque and normal erectile function (with or without the use of medications) (Level 2 Evidence, Grade B Recommendation). In both trials, men in the CCh group were shown to exhibit a $34 \%\left(-17.0 \pm 14.8^{\circ}\right)$ improvement in penile curvature, compared to $18 \%\left(-9.3 \pm 13.6^{\circ}\right)$ in the placebo group, as well as a significantly decreased PD bother score. While uncommon, reported adverse events included significant penile hematomas, injection site pain, and penile swelling. Corporal rupture, while possible was extremely rare, but often will necessitate surgical repair. ${ }^{37-39}$ Use of this technique in men with hinge defects, ventral curvature, hourglass deformities, curvature less than 30 and greater than 90 degrees has not been evaluated. ${ }^{35-36}$ Modifications and optimization of treatment protocols continue, as do studies for patients outside of the initial inclusion criteria as the are key for determining efficacy across wider PD populations. ${ }^{1,40-41}$

$\underline{\text { Verapamil }}$

In Canada at present, the use of intralesional verapamil for local treatment of PD is a commonly used agent with more than two decades of experience. The technique and first reportswere published in the mid-1990s ${ }^{42}$, and demonstrated reduction or stabilization of plaque size and improved penile deformity using a q 2 week series of injections with several modifications to treatment regimens since. ${ }^{43}$ While acknowledging that there currently exists no perfect animal model of PD,intralesional verapamil injection in PD models has shown histologic evidence of cellular changes of decreased collagen and elastin fibers and functional erectile improvement; ${ }^{43}$ ILV has been evaluated in eleven randomized designs, including two RCTs, and nine observational studies. $^{1,3,44,45}$

Despite great strides having been achieved in our understanding of the mechanism of PD development and defining the ideal local treatment regime, clinicians and researchers alike, many unanswered questions remain. It appears that the injected volume, frequency, concentration and duration of the ILV injection protocol affects outcome results, with longer treatment periods of concentrated ILV in younger men with small plaques but large curvature, to be optimal. ${ }^{46}$ Common reported adverse eventsinclude penile bruising, swelling and pain at the injection site, with excellent systemic safety. Discussion of ILV versus other treatments and the relative efficacies is difficult at best, as ILV data is predominantly focussed on patients in the earlier stages with active and evolving symptoms, varied protocols and endpoints, the lack of confirmation of delivery into target plaques (imaging guided), and the conflicting findings reported. ${ }^{3}$ Predictors of efficacy include higher dilutions of verapamil, younger age, and greater baseline curvature. ${ }^{1,45,46}$

Interferon alpha-2b

Intralesional interferon injection for PD is rarely used in Canada owing to cost and incidence of adverse events, but has been well studied with a large multicenter, placebo-controlled, 
prospective trial $^{47}$ and 8 observational studies (inclusion criteria and dosing regimens varied considerably). ${ }^{3}$ It demonstrated significant improvement in penile curvatureand decreased plaque size compared to placebo. Perhaps most importantly, a recent trial evaluated interferon alpha-2b and demonstrated that it reduced curvature without affecting penile vascular parameters, and outcome was independent of pre-treatment PD curvature or duration. ${ }^{48}$ Common adverse events include sinusitis in 40 to $100 \%$ of patients, flulike symptoms of arthralgia, fever and chills, and local effects of the injection such as bruising and swelling; symptoms are effectively treated in most cases with over-the-counter nonsteroidal anti-inflammatory agents and oral hydration. ${ }^{1,3}$ Intralesional IFN $\alpha 2 \beta$ can be utilized in men with curvature of at least $30^{\circ}$ without calcified plaques with modest efficacy (Level 2 Evidence, Grade B Recommendation).

\section{$\underline{\text { Corticosteroids }}$}

Corticosteroids are not recommended for intralesional treatment of PD (Grade C). Among the first intra-lesional agents utilised, ${ }^{49}$ marginal objective improvement was reported with unacceptable side effect profiles. Interestingly, the placebo group in some reports demonstrated similar efficacy indicating the potential role for plaque disruption as the true therapeutic agent. ${ }^{50}$ The risks associated with steroid use, (wound infection, local bruising/bleeding) coupled with limited efficacy do not support its use. ${ }^{51}$

Hyaluronic acid and botulinum toxin A (onabotulinumtoxinA)

Hyaluronic acid (HA) is a glycosaminoglycan that has been shown to regulate the immune system by decreasing inflammatory cytokines, and thus has been used to reduce inflammation and scar formation. Two retrospective studies and a more recent prospective study have shown a reduction in penile plaque size and curvature, as well as improved penile rigidity. ${ }^{52-54}$ Botulinum toxin has been shown to reduce fibrosis and scarring. A single study evaluated botulinum toxin A as a treatment for PD and has shown a positive response for safety and improvement in penile curvature, while data from further investigations is pending. ${ }^{55}$ It is far too early to make any recommendations on the use of these medications until more safety and efficacy data are available, and use should be limited to study populations with full informed patient consent.

$\underline{\text { Platelet-derived growth factors (platelet-rich plasma)/Priapius Shot }}{ }^{\mathrm{TM}}$ protocols and stem cell therapy

There is no Level 1-4 or Grade A-C evidence for PRP treatment of Peyronie's disease. Patients should be counselled regarding the lack of efficacy data. It is challenging at times to clearly communicate the difference between commercial communications (which are not Health Canada or FDA evaluated) versus either HC/FDA approved treatments versus off-label therapies with supportive peer-reviewed literature. PRP and commercial versions of such, although having an inherent appeal, are not recommended as treatments for Peyronie's disease.

Stem cell treatments for sexual disorders are steadily being introduced into clinical trials, and are particularly attractive for PD as cellular therapy offers a treatment modality that might 
reverse disease progression and cure the underlying pathophysiology. ${ }^{56}$ Studies are currently being published demonstrating the safety of intrapenile injection of autologous bone marrowand adipose tissue-derived stem cells; the clinical applications (safety and efficacy) of this approach are yet to be determined and patients may be made aware of accruing trials at www.clinicaltrials.gov if they are interested in this approach. A recently reported study of 11 men with combination of autologous stromal vascular fraction (SVF) and penileultrasound shock wave (see below) treatments illustrates the care that must be taken when interpreting designing studies and subsequent interpretation, as contemporary primary outcome measures were not utilized, and data extrapolation to clinical practice is limited. ${ }^{57}$

\section{Mechanical therapy - penile traction}

Mechanical traction and tissue expansion therapy is commonly used across many disciplines in medicine and is known to result in the alteration of connective tissue by cellular proliferation and expansion of the extracellular matrix and has gained rapid integration into the multimodal management of PD. ${ }^{1}$ Traction impact on PD cells in a mechanical strained environment results in alteration of connective tissue, demonstrating measurable changes in collagen and tissue metalloproteinase expression which are two key factors in determining plaque integrity. ${ }^{59}$ The current treatment paradigm for PD, relies on tissue remodeling for optimal outcomes following both minimally invasive treatment approaches and post-surgical results. The use of a traction device to reduce plaque/wound contracture, enhance plaque elasticity and reduce plaque size is appealing, given its low cost and general freedom from adverse effects.

Penile traction is recommended as part of PD management (Level 4 Evidence, Grade C Recommendation). While there exists a dearth of well designed randomized and controlled published studies on penile traction therapy, the existing literature does show some clear benefits. ${ }^{1,3,60}$ It appears to have some activity in correcting penile pain, curvature and maintenance of penile length, with recent reports highlighting a potential synergy with intralesional or oral agents. Hellstrom's group has recently reported that routine penile traction therapy during intralesional injection with interferon $\alpha-2 b$ for Peyronie's disease may result in a small but subjectively meaningful improvement in stretched penile length, without affecting curvature, if used for at least 3 hours a day. ${ }^{61}$ It must be noted that all studies vary in time the devices are applied, and the manner in which this is performed. For many patients, there is no defined teaching and follow-up protocol, and use is self-reported; as with any physiotherapy, a "perfect" stretch may be more useful than a prolonged traction session with the device improperly used or incomplete traction. Additional studies defining the type of device, optimal approach, duration and tension applied to the penis, remain as yet undefined. Given the low risk of adverse events, despite its reportedly modest therapeutic effect based on currently available literature, this appears to be a treatment approach that has potential for many men with PD. 


\section{Extracorporeal shockwave lithotripsy}

The role of extracorporeal shock wave therapy (ESWT) for PD has not been without controversy, as proponents for this modality have advocated for dual purposes, resolution or improvement of penile pain (evidence-based) and reduction of plaque size/reduction of penile curvature (not evidence-based at this time).

Contemporary guidelines have clearly demarcated ESWT's role in PD management, and the Committee concurs with this approach, supporting ESWT for potential penile pain improvement (Level 2 Evidence, Grade C Recommendation) based on three RCTs. ${ }^{1,362-65}$ The Committee does not support the use of ESWT for the reduction of penile curvature or plaque size (Level 2 Evidence, Grade C Recommendation), based three RCTs and one randomized study design (as well 15 observational studies). ${ }^{3,62-65}$ Outcomes are interpreted with caution due to methodological flaws, and there is anticipation of future studies clarifying ESWTs role in PD curvature resolution more clearly.

\section{Radiation therapy}

The use of radiation therapy (RT) for PD is not supported as evidenced by 8 observational studies, a clear lack of demonstrable efficacy and the potential risks of exposing patients to RT (potential moderate health risk in the setting of uncertain benefit). ${ }^{3}$

\section{Section three: Surgical management of Peyronie's disease}

\section{Overview}

The overarching goal of all PD treatments is to correct penile deformity while preserving penile length and the ability to attain and maintain an erection sufficient for satisfactory sexual intercourse. Surgical management is no exception, with reconstructive procedures allowing for potentially the most rapid, reliable, and sustained outcomes for correction of penile deformities. A single universal procedure that defines surgical standard of care or optimal approach does not exist; procedures for PD include penile plication, plaque incision/excision with grafting, and penile prosthesis implantation. ${ }^{1,3,66-73}$ Penile reconstructive procedures do not fall into the domain of all urologic surgeons and it is entirely appropriate to utilize subspecialty colleagues for PD surgical management in order to optimize patient outcomes, given the fact that expertise with all three surgical approaches portends greatest chance of satisfactory result.

Patient assessment, consent, and treatment planning

PD should be stable when surgical intervention is being considered. General criteria include a minimum of 6-12 months after disease onset, plaque stability for 3-6 months, and deformity precluding or making intercourse difficult. Moreover, there are further situations that play a part in a patient's decision making process, including such factors such as failed conservative or medical therapies, extensive penile plaque(s) from the outset, or patient preference for rapid results when disease is stable. ${ }^{66-73}$ It is not incorrect to bypass medical management, and proceed 
straight to surgery. However, the patient must clearly be aware and consented to the potential of treatment side-effects of surgery. Although pain is associated with acute phase, if persistent penile pain during erection is related to penile deformity, surgery may be considered even in the presence of said symptom. ${ }^{73}$

Evaluation of the patient should establish location, degree and direction of curvature(s), and presence of hinge or hourglass deformities as outlined in the Diagnosis subsection. It is critical to establish the PD disease parameters, as well as the presence or absence of ED, as this will dictate the surgical approaches considered and will also serve to set realistic expectations for the patient as related to the intervention. ${ }^{1,3,66-73}$ Combination of color duplex ultrasonography (CDU) with intracavernosal injection is the gold standard and recommended by the Committee as an integral part of preoperative evaluation (Level 4 Evidence, Grade C Recommendation); CDU-ICI may not be required in cases where normal rigidity is present and digital photographs at full erection delineate both extent of disease and intact erectile function. In this way, operative planning is optimized, and counseling specific to surgical approach possible. For example, given that a significant proportion of men with PD will have concurrent ED, and owing to operative risk of decreased rigidity with most approaches, grafting procedures may be avoided when erectile function is compromised and the patient counseled toward inflatable penile prosthesis (IPP) surgery.

Managing expectations for PD patients considering surgery is paramount. Given the psychological impact of PD on the patient, and often their partner, many men have depression or depressive symptoms, decreased self-esteem, and at times unattainable expectations in light of factors such as loss of penile length, ED, and sensory changes. ${ }^{15}$ Sensory alteration (decreased penile sensation) is infrequently reported and usually transient in nature, rarely effecting ejaculation and orgasm function. ${ }^{15}$ Patients must be made aware of the concept of "functionally straight" (penetrative intromission not compromised, in most cases this corresponds to residual curvature less than 20 degrees) versus completely straight (comparing to pre-PD anatomy), additional loss of length with tunical shortening approaches, and decreased sexual function (ie. ED, sensory) with any surgical procedures. ${ }^{66-76}$

Components of informed consent should include discussion of all treatment options with the patient understanding the potential for treatment complications; it is not reasonable to expect the surgeon to convey all potential risks, rather common potential side effects including persistent (failed straightening) or recurrent deformity, penile length loss (flaccid and erect), ED specifically calling out to attention decreased rigidity, sensory changes, and orgasm/ejaculation changes. In addition to this, specific advantages and drawback for treatments shortening the convex side (plication), lengthening the concave side (incision, partial excision, or excision of plaque(s)) with grafting, and IPP with adjunct procedures such as remodeling, plication or grafting are required. ${ }^{1,3,66-77}$ Finally, the patient should have an understanding of contemporary PD surgery algorithms, and how they are applied to his particular case: 
a) Plication surgery - preferred for men with adequate penile length, intact erectile function with or without pharmacotherapy, curvature that is reasonably correctable with this approach, and minimal/absent hourglass deformity causing hinging. By default these men will have penile length loss with the repair.

b) Grafting techniques - intact erectile function with severe curvature, indentation, hourglass or complex deformities, and concern or functional compromiseattributable to further length loss with plication approaches

c) Inflatable penile prosthesis (IPP) - complex penile deformities not amenable to the above techniques, presence of refractory erectile dysfunction, or patient preference

It is beyond the scope of this guideline to present a full survey of contemporary published surgical PD literature; the interested reader is guided to Nehra et al. for this type of analysis. ${ }^{3}$ All surgical PD recommendations are considered (Level 3 Evidence, Grade C Recommendation), based on over 200 observational studies cumulatively published for plication, grafting and IPP procedures. There are no RCTs for PD surgery, and interpretation is complicated by design differences, range of inclusion criteria, variance in types of surgery performed and specific outcomes measured, and range of follow-up durations. ${ }^{3,66-77}$

\section{Plication surgery}

Plication procedures represent the most common type of surgical approach for PD, and are attractive due to a high degree of curvature correction and the relatively low risk of adverse events. ${ }^{3}$ There have been no head-to-head studies of the primary types of plication surgery, named Nesbit and variants (elliptical excision of the tunica albuginea [TA] contralateral to the area of maximal curvature), Yachia (vertical tunical incision closed transversely in a HeinekeMiculicz fashion without TA tissue removal), the 16-dot or pure plications (no incision into TA, but plicated with permanent sutures using an extended Lembert-type suture), and TAP (tunica albuginea plication) procedures. ${ }^{78-84}$ For TAP, the incisions through the external longitudinal TA fibers without violation of internal circular fibers are separated by $0.5-1.0 \mathrm{~cm}$ and longitudinal fibers between the two transverse incisions are removed to reduce the bulk of the plication. ${ }^{85}$ For plication surgery, a circumcising incision may permit better exposure but carriesthe risk of preputial edema post-operatively; alternatively a midline incision may be preferred to accessthe TA for men who are uncircumcised and do not wish to undergo circumcision.

The success and satisfaction of plication varies depending on technique, but to be clear, these differences do not reflect superiority of one technique over another as direct comparisons across the observational studies cannot be made. ${ }^{2}$ Complete curvature correction rates range from $42-100 \%$ and overall satisfaction ranges from $68-100 \%$, with primary satisfaction determinants being straightening and improved sexual performance; conversely, dissatisfaction correlates topostoperative penile shortening (all tunical shortening procedures invariably decrease expansion of the TA contralateral to inelastic plaque segments), ED, pain and change of penile shape (worsening curvature 2-28\%) and sensation (wide range across studies, 24\% reported with elevation of neurovascular bundle with Nesbit and Yachia). ${ }^{1,37,783-85}$ 
Regarding penile length loss, Hudak reported $84 \%$ of men had no measureable decrease in SPL but 78\% reported perceived length reduction while Taylor and Levine reported post-surgery length loss vs objective length loss documented in $18 \%$ of these patients. ${ }^{83-85}$ Following plication, penile length should be similar to preoperative stretched flaccid length (SPL); preoperatively, it is often useful to illustrate the estimated length loss on the long side of the penis to the patient as measured during erection at CDU/ICI testing by measuring the difference in length between the long and short side of the penis. ${ }^{78}$ There has been widening use of penile traction physiotherapy device in the post-operative period;given the low risk of adverse events balanced against limited supportive literature for therapeutic benefits, it may be reasonable to consider adding traction to these patients' post-operative routines while awaiting definitive studies. ${ }^{86}$

PD plication procedures are minimally invasive, tend to preserve potency, and offer satisfactory rates of curvature correction. Drawbacks include length loss, and plication does not address and in fact may worsen existing hinge or hourglass deformities; complications may include persistent pain,persistence or recurrence of penile curvature (>30 degrees, 8-11\%), penile hematoma(0-9\%), urethral injury(0-1.4\%), palpable suture knots,and sensation loss (neurovascular bundle injury during dorsal plication). ${ }^{78}$ The type of plication utilized is dependent on surgeon and patient factors, as no procedure has proven superior to its counterparts (Level 3 Evidence, Grade C Recommendation).

\section{Grafting procedures}

Grafting technique are appropriate for the surgeon facile with the techniques and treating the PD patient with severe penile length loss, significant/severe or complex curvatures, simple curvature $>60$ degrees, large plaques, and/or hourglass deformities in the setting of maintained erectile function (Level 3 Evidence, Grade C Recommendation). Although classically regarded as "lengthening procedures", grafting does not ensure length preservation or return of lost length due to PD plaque inelasticity. Similar to plication studies, there is a vast experience of observational data encompassing more than 2500 patients in total, yet comparisons across grafting techniques and materials cannot be made to determine a superior approach. ${ }^{2,3}$ Surgeon experience, patient preference, and type of penile deformity affect the choice of graft and surgical approach used. ${ }^{76}$ The Committee strongly recommends against the use of synthetic grafts including polyester and polytetrafluoroethylene due to increased risks of infection, secondary graft inflammation causing tissue fibrosis, graft contractures, and possibility of allergic reactions (Level 3 Evidence, Grade C Recommendation). ${ }^{76,106}$

Grafting follows incision, partial excision or excision of plaque. There are three broad categories of TA plaque incision (double-Y, H-shaped, and Egydio geometric) that are made at the point/relative to the maximum curvature on the convex side of the penis, followed by placement of a graft material(autologous, allografts, xenografts, and synthetic) to repair the defect and potentially lengthen the shorter side of the penis. ${ }^{1,3,69-72,76,78,86-101}$ There is a strong trend towards minimal TA disruption, therefore favoring incision or partial excision techniques; 
partial plaque excision versus total plaque excision offers the advantage of decreasing risk of irreversible erectile tissue damage with resultant veno-occlusive permanent postoperative ED. ${ }^{76-}$ ${ }^{78,102}$ Curvature correction reliably occurs and is the most commonly reported endpoint; in a review of 88 observational study arms, improvement rates were $>80 \%$ in 64 and $>90 \%$ in $57 .{ }^{3}$ Complete deformity correction rates range from $50-98 \%$, and the satisfaction rates are highly variable from $35-51 \%{ }^{78}$ Penile shortening was observed in $7 / 11$ studies, ranging from 15.4 to $63 \%$ of subjects; grafting does not guarantee length return or length preservation. ${ }^{3}$ Post-operative care pathways are not uniform. Early return to erection is thought to be of benefit, and some experts advocate for application of external penile traction therapy to minimize loss of length once the skin incision is healed and patient can tolerated the modality. ${ }^{78}$ A retrospective study determined perception of length loss is minimal when traction therapy was utilized although dissatisfaction with penile length post-operatively remained high at almost half of surveyed patients. ${ }^{87}$

There is no universally accepted optimal graft material; the search remains for an inexpensive, readily available graft that mimics the TAs strength and elastic characteristics, has minimal morbidity (including harvest) and tissue reaction, is pliable and easy to suture, resists infection, and preserves erectile capacity. ${ }^{76,78}$ Tissue-engineered grafts may represent the future but in the meantime, currently available grafts are associated with potential complications commonly linked to significant patient dissatisfaction, the most important of which is ED and can occur in upwards of $25 \%$ of patients. ${ }^{104}$ Determinants of ED risk include age, site and severity of curvature, type of incision used, medical co-morbidities, and pre-existing erectile function. ${ }^{103-105}$ When choosing autologous graft material (saphenous vein, temporalis fascia, fascia lata, tunica vaginalis), complications at the donor tissue site and extra surgical times to harvest the grafts should be discussed with patients prior to the surgery. ${ }^{78}$

Penile sensation changes are related to freeing of the dorsal neurovascular bundle (NVB), extent of dissection required to completely dissect the area of the plaque, and post-operative inflammation and fibrosis at the graft sites; although the majority of surgeons approach the NVD laterally in contemporary practice, a medial approach may be warranted based on surgeon preference and experience, as well as patient factors. The decrease or loss of sensation is reported to by $5 \%$ or less by most studies, although rates range up to $25 \%$. $^{3}$

Patient satisfaction rates were reported in 12/88 studies, and ranged from $40.9 \%$ to 93.3\%, with eight of these above $80 \%$; partner satisfaction was only captured by 4 groups, and ranged from $72 \%$ to $100 \% .{ }^{1,3}$ Complex penile reconstruction for $\mathrm{PD}$, with grafting or IPP is demanding surgery and is most likely to offer best outcomes when performed by experienced reconstructive and prosthetic surgeons facile with multiple techniques and availability of surgery-specific resources needed to address the PD. ${ }^{1}$ 


\section{Inflatable penile prosthesis}

IPP remains the gold standard treatment for PD requiring surgery and occurring concurrently with refractory erectile dysfunction, and is appropriate for severe deformity refractory to nonsurgical management or failed plication/grafting, and with profound penile instability (buckling or hinge). ${ }^{77,78}$ The use of inflatable devices for PD is advised (Level 3 Evidence, Grade C Recommendation) and the surgeon must be able to, and have resources available for, placement of the IPP and additional procedures which are frequently required to ensure a satisfactory surgical outcome include manual modeling, plication, plaque-releasing incision(s), and grafting if TA defect size confers risk of herniation (commonly used cut-off is two cm). ${ }^{1,3,77,78}$ PD deformity correction rates with penile prosthesis implantation range from $84-100 \% .{ }^{78}$ Review of 43 observational studies yields curvature data for 26 datasets; all report curvature improvement of greater than $80 \%$. ${ }^{3}$ Satisfaction rates for general etiologies range from $91-100 \%$, while men with PD are somewhat lower that non-PD patients (79\%) although this is based on older data, and prospective study results are due shortly. ${ }^{77,78}$ Consent for PD patients receiving a penile implant includes discussingrisk for prosthesis infection, persistent penile shortening or curvature, diminished sensitivity, and mechanical device failure or difficulties.

\section{Section four: Congenital penile curvature}

Congenital penile curvature (CPC) is a uniquely different condition from Peyronie's disease with distinct evaluation and treatment approaches. CPC results from disproportionate development of the tunica albuginea of the corporal bodies and is not associated with urethral malformation. ${ }^{108}$ Patients typically present younger, often in their late teens and twenties (see Table 1). Although the condition is congenital, often patients first appreciate the curvature to their penis as they complete puberty and become concerned about initiating sexual activity. ${ }^{3,108}$ The patient gives a history of lifelong curvature, without hinge or complex deformity, and there is an absence of underlying palpable plaque or induration. ${ }^{1} \mathrm{~A}$ proportion of boys will present to their pediatric urologist with CPC, and the data remains formative as to the optimal timing for surgical intervention in this group. ${ }^{109-111}$

A focused history and physical examination establishes the diagnosis of CPC. Unlike Peyronie's disease, congenital penile curvatures are most commonly ventral curvatures, with erectile function and rigidity typically normal, and with preserved penile length. Limitations in sexual function are typically psychological or related to the physical curvature interfering with penetration. ${ }^{1,3,108}$ Untreated CPC may confer worse penile perception scores, mentally unhealthy days, and increased difficulty with intercourse secondary to curvature compared with men without curvature. $^{112}$

Treatment is surgical and is offered to patients whose CPC significantly interferes with satisfactory sexual relations of the patient or partner. There is no role for medical management of the CPC deformity (Level 3 Evidence, Grade C Recommendation). Plication is used almost exclusively with satisfactory curve correction rates in the order of 67-97\%, although there is not 
a gold standard surgical technique preferred over another as there remains an absence of comparable outcomes literature .1,3,108, 111 


\section{References}

1. Chung E, Ralph D, Kadioglu A, et al. Evidence-based management guidelines on Peyronie's Disease. J Sex Med 2016; 13:905-923.

2. Dahm P, Jung JH, Bodie J. Moving from consensus- to evidence-based clinical practice guidelines for Peyronie's disease. J Sex Med 2017; 14:170-171.

3. Nehra A, Alterowitz R, Culkin DJ, et al. Peyronie's Disease: AUA Guideline. J Urol 2015; 194(3):745-53.

4. Bella AJ, Greenfield JM (Chile KA editor). Peyronie's Disease: Epidemiology, Pathophysiology and Evaluation. AUA Core Curriculum, American Urological Association Education and Research Inc.; 2016. https://university.auanet.org/core_topic.cfm?coreid=103. Accessed July 302017.

5. Mulhall JP, Schiff J, Guhring P. An analysis of the natural history of Peyronie's disease. J Urol 2006;175(6):2115-8.

6. Berookhim BM, Choi J, Alex B, et al. Deformity stabilization and improvement in men with untreated Peyronie's disease.BJU Int. 2014;113(1):133-6.

7. Grasso M, Lania C, Blanco S, et al. The natural history of Peyronie's disease. Arch Esp Urol 2007; 60(3):326-31.

8. Bella AJ, Lee JC, Carrier S, et al. 2015 CUA Practice guidelines for erectile dysfunction. Can Urol Assoc J 2015;9(1-2):23-29.

9. Bella AJ, Sener A, Foell K, Brock GB.Nonpalpable scarring of the penile septum as a cause of erectile dysfunction: an atypical form of Peyronie's disease. J Sex Med 2007;4(1):226-30.

10. Brant WO, Bella AJ, Garcia MM. Isolatedseptal fibrosis or hematoma--atypical Peyronie's disease? J Urol 2007;177(1):179-182.

11.Rosen R, Catania J, Lue T, et al. Impact of Peyronie's disease on sexual and psychosocial functioning: qualitative findings in patients and controls. J Sex Med 2008;5:1977-84.

12. Davis SN, Ferrar S, Sadikaj G, et al. Female partners of men with Peyronie's disease have impaired sexual function, satisfaction, and mood, while degree of sexual interference is associated with worse outcomes. J Sex Med 2016;13(7):1095-103.

13. Farrell MR, Corder CJ, Levine LA. Peyronie's disease among men who have sex with men: characteristics, treatment, and psychosocial factors. J Sex Med 2013;10(8):2077-83.

14. Smith JF, Walsh TJ, Conti SL, et al. Risk factors for emotional and relationship problems in Peyronie's disease. J Sex Med 2008;5(9):2179-84.

15. Nelson CJ, Mulhall JP. Psychological impact of Peyronie’s disease: a review. J Sex Med 2013;10(3):653-60.

16. Nelson CJ, Diblasio C, Kendirci M, et al. The chronology of depression and distress in men with Peyronie’s disease. J Sex Med 2008 Aug;5(8):1985-90.

17. Davis S, Ferrar S, Sadikaj G, et al. Shame, catastrophizing, and negative partner responses are associated with lower sexual and relationship satisfaction and more negative affect in men with Peyronie’s disease.J Sex Marital Ther 2016 Feb 2:1-13. 
18. Walsh TJ, Hotaling JM, Lue TF, et al. How curved is too curved? The severity of penile deformity may predict sexual disability among men with Peyronie's disease. Int J Impot Res 2013 May;25(3):109-12.

19. Goldstein I, Hartzell R, Shabsigh R. The impact of Peyronie's disease on the patient: gaps in our current understanding. J Sex Marital Ther 2016;42(2):178-90.

20. Coyne KS, Currie BM, Thompson CL, et al. Responsiveness of the Peyronie’s Disease Questionnaire (PDQ). J Sex Med 2015 Apr;12(4):1072-9.

21. Hellstrom WJ, Feldman WJ, Rosen RC, et al. Bother and distress associated with Peyronie's disease: validation of the Peyronie's Disease Questionnaire. J Urol 2013 Aug; 190(2):627-34. 22. Richard PO, Violette PD, Jewett MAS, et al. CUA guideline on the management of cystic renal lesions. Can Urol Assoc J 2017;11:E66-73.

23.Hatzimouratidis K, Eardley I, Giuliano F, et al; EAU guidelines on penile curvature. Eur Urol 2012;62:543-552.

24. Shindel AW, Sweet G, Thieu W, et al. Prevalence of Peyronie's Disease-Like Symptoms in Men Presenting with Dupuytren Contractures. Sex Med 2017 doi 10.1016/J.sexm.2016.06.001 epub ahead of print 25. Chung E, DeYoung L, Brock GB. The role of PDE5 inhibitor in penile septal scar remodeling: Assessment of clinical and radiological outcomes. J Sex Med 2011;8:1472-1477. 26. Smith JF, Brant WO, Fradet V, et al Penile sonographic and clinical characteristics in men with Peyronie's disease. J Sex Med 2009;6:2858-2867.

27. Bekos A, Arvaniti M, Hatzimouratidis K, et al. The natural history of Peyronie's disease: an ultrasonography-based study.Eur Urol 2008;53(3):644-50.

28. Greenfield JM, Shah SJ, Levine LA. Verapamil versus saline in electromotive drug administration for Peyronie's disease: a double-blind, placebo controlled trial. J Urol 2007;177:972-975.

29. Kokab A, Wylie K, Allen P, et al. Structured self-rated response to iontophoresis with verapamil and dexamethasone in Peyronie's disease. Adv Urol 2014;2014;957013.

30. Martin, D. J., Badwan, K., Parker, M. et al.: Transdermal application of verapamil gel to the penile shaft fails to infiltrate the tunica albuginea. J Urol 2002;168:2483-2485.

31. Fitch, W. P., 3rd, Easterling, W. J., Talbert, R. L. et al.: Topical verapamil HCl, topical trifluoperazine, and topical magnesium sulfate for the treatment of Peyronie's disease--a placebocontrolled pilot study. J Sex Med 2007;4: 477-484.

32. Russell S, Steers W, McVary KT Systematic evidence-based analysis of plaque injection therapy for Peyronie's disease. Eur Urol 2007;51: 640-647.

33. Gelbard M, Walsh R, Kaufman J. Collagenase for peyronie's disease experimental studies.Urol Res 1982;10:135-140.

34. Gelbard M, Lindner A, Kaufman J. The use of collagenase in the treatment of Peyronie's disease. J Urol 185;134:280-283. 
35. Gelbard M, Goldstein I, Hellstrom WK et al. Clinical efficacy, safety and tolerability of collagenase clostridium histolyticum for the treatment of Peyronie's disease in 2 large doubleblind, randomized, placebo controlled phase 3 studies. J Urol 2013;190:199-20.

36. Lipshultz LI, Goldstein I, Seftel AD et al. Clinical efficacy of collagenase clostridium histolyticum in the treatment of Peyronie's disease by subgroup: Results from 2 large doubleblind, randomized, placebo controlled phase III studies. BJU Int 2015;116:650-656.

37. Levine LA, Cuzin B, Mark S et al. Clinical safety and effectiveness of collagenase Clostridium histolyticum injection in patients with Peyronie's disease: A phase 3 open-label study. J Sex Med 2015; 12: 248-258.

38. Hobaugh C, Pastuszak AW. Serious complications of collagenase clostridium histiolyticum injection for Peyronie's disease: more than meets the eye. Transl Androl Urol 2017;6(2):320-21. 39. Hellstrom WJG, Tan RBW, Liu G. Safety Profile of Collagenase Clostridium Histolyticum Stratified by Degree of Penile Curvature in Patients with Peyronie's Disease. Urology 2017;17:30477-6.

40. Abdel Raheem A, Capece M, Kalejaiye O, et al. Safety and effectiveness of collagenase clostridium histiolyticum in the treatment of Peyronie's disease using a new modified shortened protocol. BJU Int 2017; Jun 14 doi:10.1111/bju.13932 epub ahead of print

41. Goldstein I, Knoll LD, Lipshultz LI, et al. Changes in the effects of Peyronie's disease after treatment with collagenase clostridium histiolyticum: Male patients and their female partners. Sex Med 2017;5(2):e124-e130.

42. Levine LA, Merrick PF, Lee RC. Intralesional verapamil injection for the treatment of Peyronie's disease. J Urol 1994; 151: 1522-1524.

43. Rehman J, Benet A, Melman A. Use of intralesional verapamil to dissolve Peyronie's plaque: A long-term single blind study. Urology 1998;51:620-626.

44.Chung E, Garcia F, Young LD et al. A comparative study of the efficacy of intralesional verapamil versus normal saline injection in a novel Peyronie's animal model: Assessment of immunohistopathological changes and erectile function outcome. J Urol 2013;189:380-384. 45.Moskovic DJ, Alex B, Choi JM et al. Defining predictors of response to intralesional verapamil injection therapy for Peyronie's disease. BJU Int 2011;108:1485-1489.

46. Cavalini G, Modenini F, Vitali G. Open preliminary randomized prospective clinical trial of efficacy and safety of three different verapamil dilutions for intraplaque therapy of Peyronie's disease. Urology 2007;69:950-954.

47. Hellstrom WJ, Kendirci M, Matern R et al. Single-blind, multicentre, placebo controlled, parallel study to assess the safety and efficacy of intralesional interferon alpha-2B for minimally invasive treatment for Peyronie's disease. J Urol. 2006;176:394-398.

48. Trost LW, Ates E, Powers $M$ et al. Outcomes of intralesional interferon- $\alpha 2 \mathrm{~B}$ for the treatment of Peyronie's disease. J Urol 2013;190:2194-2199.

49. Teasley GH. Peyronie's disease: A new approach. J Urol 1954;71:611-614. 
50. Cipollone G, Nicolai M, Mastroprimiano G et al. Betamethasone versus placebo in Peyronie's disease. Arch Ital Androl 1998;70:165-168.

51. Desanctis PN and Furey CA Jr. Steroid injection therapy for Peyronie's disease: A 10-year summary and review of 38 cases. J Urol 1967;97:114-116.

52. Favilla V, Russo GI, Zucchi A et al. Evaluation of intralesional injection of hyaluronic acid compared with verapamil in Peyronie's disease: preliminary results from a prospective, doubleblinded, randomized study. Andrology 2017;5:771-775.

53. Zucchi A, Costantini E, Cai T, et al. Intralesional Injection of Hyaluronic Acid in Patients Affected With Peyronie's Disease: Preliminary Results From a Prospective, Multicenter, Pilot Study. Sex Med 2016;4:e83-88.

54. Gennaro R, Barletta D, Paulis G.Intralesional hyaluronic acid: an innovative treatment for Peyronie's disease. Int Urol Nephrol 2015;47:1595-602.

55. Muñoz-Rangel CA, Fernandez-Vivar E, Bañuelos-Gallo RA, et al. Minimally Invasive Therapy Using Intralesional OnabotulinumtoxinA in Peyronie's Disease. Urol J 2015;12:210510.

56.Soebadi MA, Milenkovic U, Weyne E et al. Stem Cells in Male Sexual Dysfunction: Are We Getting Somewhere? Sex Med Reviews 2017;5:222-235.

57. Lander EB, Berman MH, See JR.Stromal Vascular Fraction Combined with Shock Wave for the Treatment of Peyronie's Disease. Plast Reconstr Surg Glob Open 2016;4:e631.

58. Chung E, Brock GB. Penile traction therapy and Peyronie's disease: A state of art review of the current literature. Ther Adv Urol 2013;5: 59-65.

59. Chung E, De Young L, Solomon M, et al. Peyronie's disease and mechanotransduction: An in vitro analysis of the cellular changes to Peyronie's disease in a cell-culture strain system. J Sex Med 2013;10: 1259-1267.

60. Abern MR, Larsen S, Levine LA.Combination of penile traction, intralesional verapamil, and oral therapies for Peyronie's disease. J Sex Med 2012;9:288-95.

61. Yafi FA, Pinsky MR, Stewart C, et al. The effect of duration of penile traction therapy in patients undergoing intralesional injection therapy for Peyronie's disease. J Urol 2015;194: 754758.

62. Chitale S, Morsey M, Swift L et al. Limited shock wave therapy vs sham treatment in men with Peyronie's disease: results of a prospective randomized controlled double-blind trial. BJU Int 2010;106: 1352-56.

63. Hatzichristodoulou G, Meisner C, Gschwend JE et al. Extracorporal shockwave therapy in Peyronie's disease: results of a placebo-controlled, prospective, randomized, single-blind study. J Sex Med 2013;10:2815-21.

64. Palmieri A, Imbimbo C, Longo N et al. A first prospective, randomize, double-blind, placebo-controlled clinical trial evaluating extracorporeal shock wave therapy for the treatment of Peyronie's disease. Eur Urol 2009;56:363-69. 
65. Palmieri A, Imbimbo C, Creta M, et al. Tadalafil once daily and extracorporeal shock wave therapy in the management of patients with Peyronie's disease and erectile dysfunction: results from a prospective randomized trial. Int J Androl 2012;35:2190.

66. Kendirci M, Hellstrom WJ. Critical analysis of surgery for Peyronie's disease. Curr Opin Urol 2004;14:381-8.

67. Ralph D, Gonzalez-Cadavid N, Mirone V, Perovic S, Sohn M, Usta M et al. The management of Peyronie's disease: evidence-based 2010 guidelines. J Sex Med 2010;7:2359-74. 68. Kadioglu A, Kucukdurmaz F, Sanli O. Current status of the surgical management of Peyronie's disease. Nat Rev Urol 2011;8:95-106.

69. Hatzimouratidis K, Eardley I, Giuliano F, et al. EAU guidelines on penile curvature. Eur Urol 2012;62:543-52.

70. Levine LA, Burnett AL. Standard operating procedures for Peyronie's disease. J Sex Med 2013;10:230-44.

71. Levine LA, Larsen SM. Surgery for Peyronie's disease. Asian J Androl 2013;15:27-34.

72. Segal RL, Burnett AL. Surgical Management for Peyronie's Disease. World J Mens Health 2013;31:1-11.

73. Zaid UB, Alwaal A, Zhang X, Lue TF. Surgical management of Peyronie's disease. Curr Urol Rep 2014;15:446.

74. Matsushita K, Stember DS, Nelson CJ et al. Concordance between patient and physician assessment of the magnitude of Peyronie's disease curvature. J Sex Med 2014;11:205-210. 75. Taylor FL, Levine LA. Surgical correction of Peyronie's disease via tunica albuginea plication or partial plaque excision with pericardial graft: Long-term follow up. J sex Med 2008;5:2221-2228.

76. Hatzichristodoulou G, Osmonov D, Kubler H, et al. Contemporary review of grafting techniques for the surgical treatment of Peyronie's disease. Sex Med Rev 2017;S20500521(17)30008-2 Feb 26 (epub ahead of print)

77. Levine LA, Becher E, Bella A, et al. Penile Prosthesis Surgery: Current Recommendations From the International Consultation on Sexual Medicine.J Sex Med 2016;13(4):489-518.

78. Levine LA, Mulhall JP, Wang R (Chile KA editor). Peyronie's Disease Surgical Management. AUA Core Curriculum, American Urological Association Education and Research Inc.; 2016. https://university.auanet.org/core_topic.cfm?coreid=103. Accessed July 302017. 79. Lemberger RJ, Bishop MC, Bates CP. Nesbit's operation for Peyronie's disease. Br J Urol 1984;56: 721-3.

80. Yachia D. Modified corporoplasty for the treatment of penile curvature. J Urol 1990;143:802.

81. Gholami SS, Lue TF. Correction of penile curvature using the 16-dot plication technique: a review of 132 patients. J Urol 2002;167:2066-9.

82. Brant WO, Bella AJ, Lue TF. 16-dot procedure for penile curvature. J Sex Med 2007;4:277280. 
83. Taylor FL, Levine LA. Surgical correction of Peyronie's disease via tunica albuginea plication or partial plaque excision with pericardial graft: long-term follow up. J Sex Med 2008;5:2221-8.

84. Rehman J, Benet A, Minsky LS, et al. Results of surgical treatment for abnormal penile curvature: Peyronie's disease and congenital deviation by modified Nesbit plication (tunical shaving and plication). J Urol 1997;157:1288-91.

85. Hudak SJ, Morey AF, Adibi M, et al. Favorable patient reported outcomes following penile plication for wide array of Peyronie’s deformities. J Urol 2013; 189:1019-24.

86. Gelbard MK, Hayden B. Expanding contractures of the tunica albuginea due to Peyronie's disease with temporalis fascia free grafts. J Urol 1991;145:772-6.

87. Rybak J, Papagiannopolous D, Levine L. A retrospective comparative study of traction therapy vs. no traction following tunica albuginea plication or partial excision and grafting for Peyronie's disease: measured lengths and patient perceptions. J Sex Med 2012;9:2396-403. 88. Levine LA, Estrada CR. Human cadaveric pericardial graft for the surgical correction of Peyronie's disease. J Urol 2003;170:2359-62.

89. El-Sakka AI, Rashwan HM, Lue TF. Venous patch graft for Peyronie's disease. Part II: outcome analysis. J Urol 1998;160:2050-3.

90. Kovac JR, Brock GB. Surgical outcomes and patient satisfaction after dermal, pericardial, and small intestinal submucosal grafting for Peyronie's disease. J Sex Med 2007;4:1500-8.

91. Knoll LD. Use of small intestinal submucosa graft for the surgical management of Peyronie's disease. J Urol 2007;178:2474-8.

92. Breyer BN, Brant WO, Garcia MM, Bella AJ, Lue TF. Complications of porcine small intestine submucosa graft for Peyronie's disease. J Urol 2007;177:589-91.

93. Chung E, Clendinning E, Lessard L, Brock G. Five-year follow-up of Peyronie's graft surgery: outcomes and patient satisfaction. J Sex Med 2010;8:594-600.

94. Egydio PH, Lucron AM, Arap S. A single relaxing incision to correct different types of penile curvature: Surgical technique based on geometric principles. BJU Int 2004;94:1147-1157. 95. Miranda AF, Sampaio FJ. A geometric model of plaque incision and graft for Peyronie's diseasewith geometric analyses of different techniques. J Sex Med 2014;11:1546-1553. 96.Adeniyi AA, Goorney SR, Pryor JP, Ralph DJ. The Lue procedure: an analysis of the outcome in Peyronie's disease. BJU Int 2002;89:404-8.

97. Kalsi J, Minhas S, Christopher N, Ralph D. The results of plaque incision and venous grafting (Lue procedure) to correct the penile deformity of Peyronie's disease. BJU Int 2005;95:1029-33.

98. Montorsi F, Salonia A, Maga T, et al. Evidence based assessment of long-term results of plaque incision and vein grafting for Peyronie's disease. J Urol 2000;163:1704-8.

99. Sansalone S, Garaffa G, Djinovic R, et al. Long-term results of the surgical treatment of Peyronie's disease with Egydio's technique: a European multicentre study. Asian J Androl 2011;13:842-5. 
100. Yafi FA, Hatzichristodoulou G, DeLay KJ,Hellstrom WJ.Review of Management Options for Patients With Atypical Peyronie's Disease.Sex Med Rev 2017;5(2):211-221.

101. Gaffney CD, Pagano MJ, Weinberg AC, et al. Lengthening strategies for Peyronie's disease.Transl Androl Urol 2016;5(3):351-62.

102. Wimpissinger F, Parnham A, Gutjahr G, et al. 10 Years' Plaque Incision and Vein Grafting for Peyronie's Disease: Does Time Matter?J Sex Med 2016;13(1):120-8.

103.Taylor FL, Abern MR, Levine LA. Predicting erectile dysfunction following surgical correction of Peyronie's disease without inflatable penile prosthesis placement: vascular assessment and preoperative risk factors. J Sex Med 2012;9:296-301.

104. Levine LA, Greenfield JM, Estrada CR. Erectile dysfunction following surgical correction of Peyronie's disease and a pilot study of the use of sildenafil citrate rehabilitation for postoperative erectile dysfunction. J Sex Med 2005;2:241-247.

105. Kozacioglu Z, Degirmenci T, Gunlosoy B et al. Effect of tunical defect size after Peyronie's plaque excision on post-operative erectile function. Do centimeters matter? Urology 2012;80:1051-1055.

106. Kadioglu A, Sanli O, Akman T, et al. Graft materials in Peyronie's disease surgery: A comprehensive review. J Sex Med 2007;4:581-595.

107. Trost LW, Munarriz R, Wang R, et al. External Mechanical Devices and Vascular Surgery for Erectile Dysfunction.J Sex Med 2016;13(11):1579-1617.

108. Makovey I, Higuchi TT, Montague DK, et al. Congenital penile curvature: update and management. Curr Urol Rep. 2012;13(4):290-7.

109. Ozkuvanci Ü, Ziylan O, Dönmez MI, et al. An unanswered question in pediatric urology: the post pubertal persistence of prepubertal congenital penile curvature correction by tunical plication.Int Braz J Urol. 2017 Jun 14 epub ahead of print.

110. McQuaid JW, Johnson EK, Andrews E, et al. The Efficacy of Congenital Penile Curvature Repair in Preadolescent Males: Early Outcomes.Urology 2016;92:95-9.

111. Kadirov R, Coskun B, Kaygisiz O, et al. Penile Plication With or Without Degloving of the Penis Results in Similar Outcomes.Sex Med. 2017 Jul 13. epub ahead of print

112. Menon V, Breyer B, Copp HL, et al. Do adult men with untreated ventral penile curvature have adverse outcomes?J Pediatr Urol. 2016;12:31.e1-7. 


\section{Figures and Tables}

Fig. 1. Treatment algorithm.

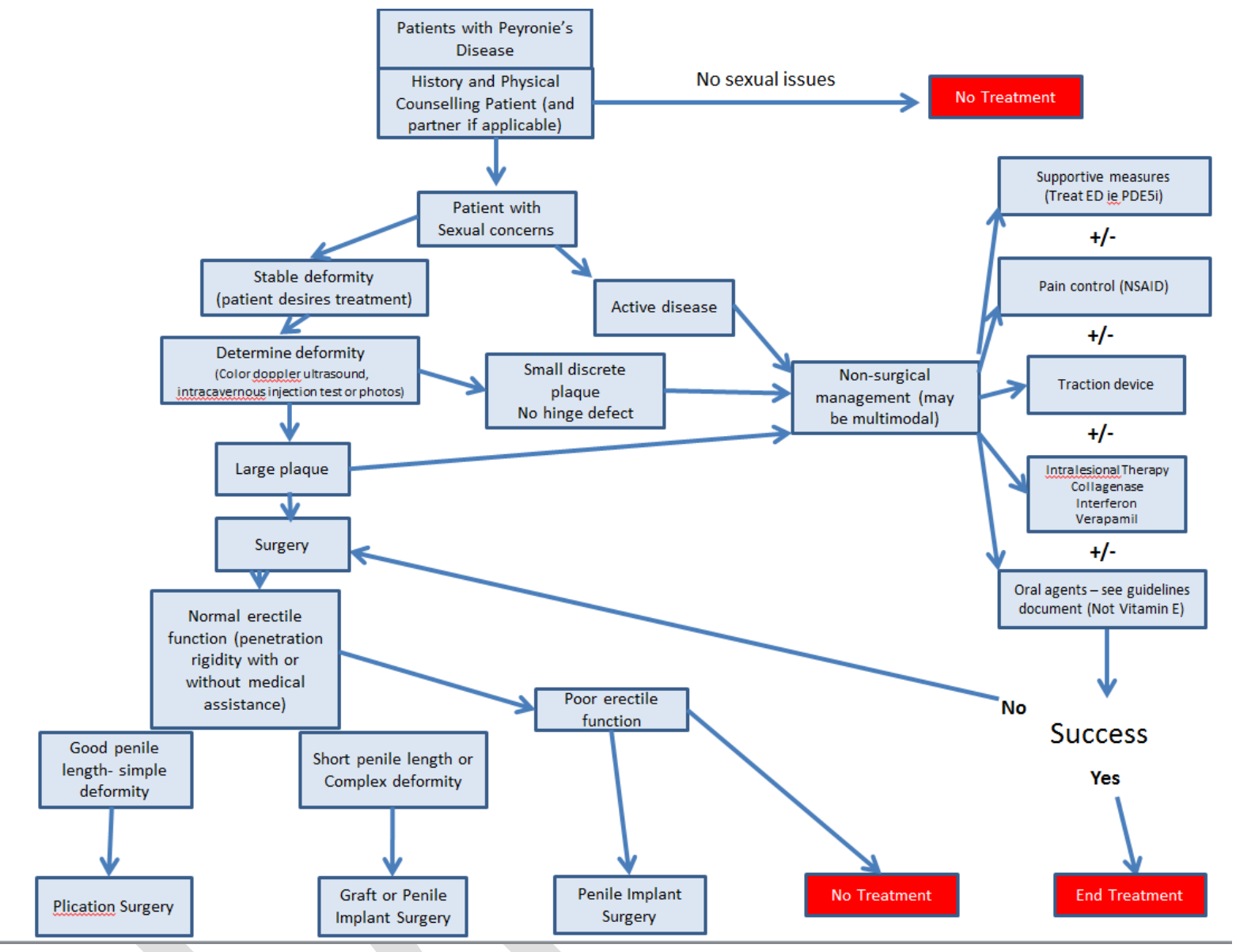

